\title{
Self-Assembled Silica-Carbonate Structures and Detection of Ancient Microfossils
}

\author{
J. M. García-Ruiz, ${ }^{1}$ S. T. Hyde, ${ }^{2}$ A. M. Carnerup, ${ }^{2}$ \\ A. G. Christy, ${ }^{2 *}$ M. J. Van Kranendonk, ${ }^{3}$ N. J. Welham ${ }^{2} \dagger$
}

We have synthesized inorganic micron-sized filaments, whose microstucture consists of silica-coated nanometer-sized carbonate crystals, arranged with strong orientational order. They exhibit noncrystallographic, curved, helical morphologies, reminiscent of biological forms. The filaments are similar to supposed cyanobacterial microfossils from the Precambrian Warrawoona chert formation in Western Australia, reputed to be the oldest terrestrial microfossils. Simple organic hydrocarbons, whose sources may also be abiotic and indeed inorganic, readily condense onto these filaments and subsequently polymerize under gentle heating to yield kerogenous products. Our results demonstrate that abiotic and morphologically complex microstructures that are identical to currently accepted biogenic materials can be synthesized inorganically.

Evidence for the oldest known fossils has rested with reported microfossil filaments from the 3.49-billion-year-old Dresser Formation (1) and the slightly younger (3.465 billion years) chert in the Apex basalt (2-5), in the Warrawoona Group of the Pilbara craton in Western Australia. Optical and electron microscopic studies confirmed the presence of complex segmented filaments in these rocks that are similar in morphology to modern cyanobacteria (1-5). Raman spectra of the Dresser and Apex filaments reveal the presence of carbonaceous residue $(6,7)$. We demonstrated the formation and growth of filamentous materials under alkaline, mildly hydrothermal conditions, whose morphologies and Raman spectra closely mimic those of the Warrawoona microfossils.

We obtained crystal aggregates by mixing a water-soluble barium salt with alkaline sodium silicate solutions at ambient temperature and pressure, at a $\mathrm{pH}$ ranging from 8.5 to 11 . The $\mathrm{Na}$ and $\mathrm{Si}$ concentrations in silica solution reaction mixtures were $\sim 7.5$ and $9 \mathrm{mM}$, respectively. Most of the silicate in our solutions was likely to be in the form of dimeric $\mathrm{Si}_{2} \mathrm{O}(\mathrm{OH})_{6}^{-}$ ions (8). Reactions were typically carried out in diffusive contact with the ambient atmosphere, so that $\mathrm{CO}_{2}$ dissolution was significant, because $\mathrm{HCO}_{3}{ }^{-}$and $\mathrm{CO}_{3}{ }^{2-}$ anions formed under the

${ }^{1}$ Instituto Andaluz de Ciencias de la Tierra, Consejo Superior de Investigaciones Científicas Universidad de Granada, Facultad de Ciencias, Campus de Fuentenueva 18002, Granada, Spain. ${ }^{2}$ Department of Applied Mathematics, Research School of Physical Sciences, Australian National University, Canberra, ACT 0200, Australia. ${ }^{3}$ Geological Survey of Western Australia, 100 Plain Street, East Perth, Western Australia 6004, Australia.

*Present address: Department of Geology, Australian National University, Canberra, ACT, Australia.

$\dagger$ Present address: Department of Minerals Science, Murdoch University, Perth, Western Australia. alkaline conditions (9). The increasing carbonate concentration provoked the precipitation of witherite crystallites (barium carbonate). Precipitate morphologies varied depending on the $\mathrm{pH}$, temperature, and concentration of the reacting solution (10-12). We focus here on aggregates formed in sols made of $10 \mathrm{mM} \mathrm{BaCl}_{2}$ and $8.3 \mathrm{mM} \mathrm{SiO}_{2}$ at room temperature (13), although similar results were obtained from highly viscous gels. Above $\mathrm{pH}$ values of 10 , micrometer- to millimeter-sized cardioidshaped sheets emanated from a central convoluted mass of material. Optical photomicro- graphs collected with plane-polarized light and cross-polarized filters show a black Maltese cross pattern of extinction contours radiating from a unique center on each sheet. This pattern indicates continuous radial variation of the orientation of the witherite crystal axes (Fig. 1). We also observed some secondary sheets, seeded from cusps at the edge of a parent sheet.

Many sheets developed scrolled lips around the rim. These were particularly evident where two oppositely scrolled sections of sheet rim converged to form a cusp, from which ropelike double-helical filaments often emerged (Fig. 1).

Filament diameters typically varied between 5 and $20 \mu \mathrm{m}$, depending on their form (Fig. 2, A to D). They could extend up to several millimeters in length and, like the sheets, were clearly visible in the optical microscope. Approximately equal numbers of right- and lefthanded helices were found within a single experiment; indeed, in some cases, both enantiomers radiated from a single sheet. Pairs of scrolls within a single rope may overlap each other to the extent that only a single helical wind is discernible. The resulting structures are typically fatter than other helical patterns, although filaments of uniform size are not uncommon in individual batches if growth is arrested by removal from the parent solution.

Field-emission scanning electron micrographs (FESEMs) and transmission electron microscopy (TEM) of the sheets and filaments revealed them to be composed of densely packed colloidal rods, typically 400 $\mathrm{nm}$ in length and $40 \mathrm{~nm}$ in diameter (Fig. 1D)
Fig. 1. Witherite-silica aggregates. (A) Optical micrograph of a flat selfassembled witheritesilica aggregate, viewed under crossed polarizers. The aggregate consists of an assembly of connected cardioidal sheets. The cruciform extinction pattern within each sheet is due to radial orientation of the crystals. The aggregate grows radially, with distinct growth bands (delineated by yellow lines). Cardioids often contain marginal cusps (arrows), from which ropelike filaments emanate. (Inset) Optical micrograph of a cusp, showing scrolled lips. (B to D) Zoom sequence of FESEMs of alkali-leached sheets (with silica removed and witherite remaining), showing the orientational ordering of witherite crystallites. Scale bar in (B), $120 \mu \mathrm{m}$; in (C), $6 \mu \mathrm{m}$; in (D), $500 \mathrm{~nm}$.
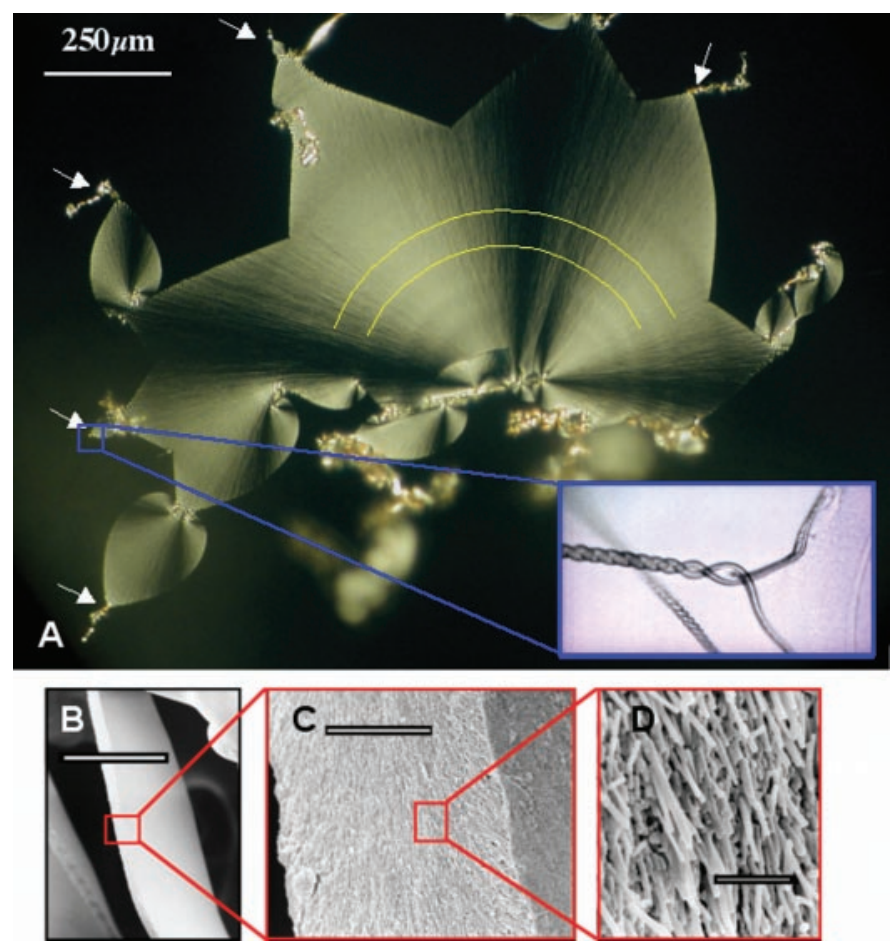
(13). Comparison of untreated specimens with those that had undergone selective dissolution of the silica (by leaching of the aggregates in dilute $\mathrm{NaOH}$ solution) showed that the rods consisted of pseudohexagonal twinned witherite crystallites (standard aragonite twin morphology), either as single crystallites or subparallel crystal aggregates, interspersed and coated with silica (table S1). Optical properties and electron diffraction showed that the long direction of the rods coincided with the pseudohexagonal $c$ axis of witherite. Orientational ordering was clear at the submicron scale, with a radial texture in both sheet and helical aggregates that is consistent with a continuous and slowly varying twist of the rod director with respect to relative position in the aggregate. X-ray diffraction patterns of sheets and helices confirmed that witherite was the only crystalline phase, and some reflections appeared as arcs rather than spots or complete rings. This unusual feature indicates a restricted range of crystal orientations, consistent with the presence of orientational order and the absence of translational order between the witherite nanocrystals within a single particle. These selfassembled aggregates are inorganic analogs of chiral liquid crystals, reminiscent of cholesteric and blue-phase mesophases (14).

These self-assembled crystal aggregates display specific reproducible morphologies, irre- spective of the gravitational orientation or rheological properties of the crystallizing medium. Their growth mechanism differs from that of conventional silica (chemical) gardens. The latter are noncrystalline metal silicate hydrate structures with morphology governed by the interaction between a chemical reaction and osmotic- and buoyancy-driven convective flow (15). Packing of the monodisperse colloidal rods within the self-assembled aggregates accompanies the growth of the curvilinear sheets and helical filaments reported here, with a relative twist between adjacent rods. The origin of that twist and the transition from sheets to helical ropes are likely to result from competing colloidal interactions between the rods, dependent on their aspect ratio and the charge distribution over their surface, set by the adsorbed silica species and charges on the witherite crystallite faces. The resulting inorganic aggregates are complex self-assemblies, displaying a hierarchy of structural order at various length scales that is similar to that of biomineral assemblies (16): ranging from the translational order in the individual witherite microcrystallites, observed in TEM micrographs, to the largerscale material, characterized by orientational order and evident from FESEM images, resulting in the curvilinear forms that are visible with optical microscopy. Selective removal of the witherite by immersion in dilute acid is also possible, leaving a hol-
Fig. 2. Comparison of synthetic filaments with purported ancient microfossils. (A to D) FESEM images of inorganic in vitro filaments. (A), (B), and (D) As-prepared filaments, containing silica and witherite. [Adapted with permission from (12).] (C) Bare barium carbonate (witherite) crystallite aggregate after dissolution of silica in mild alkaline solution. (D) Silica skin, coating the exterior of the aggregates. ( $\mathrm{E}$ and $\mathbf{F}$ ) Microfilaments found in the Warrawoona chert. [(E) Adapted with permission from (6); (F) adapted with permission from (19).] (G to I) Optical micrographs of synthetic filaments, showing the progressive dissolution of the solid (witherite) interior of the biomorphs in dilute

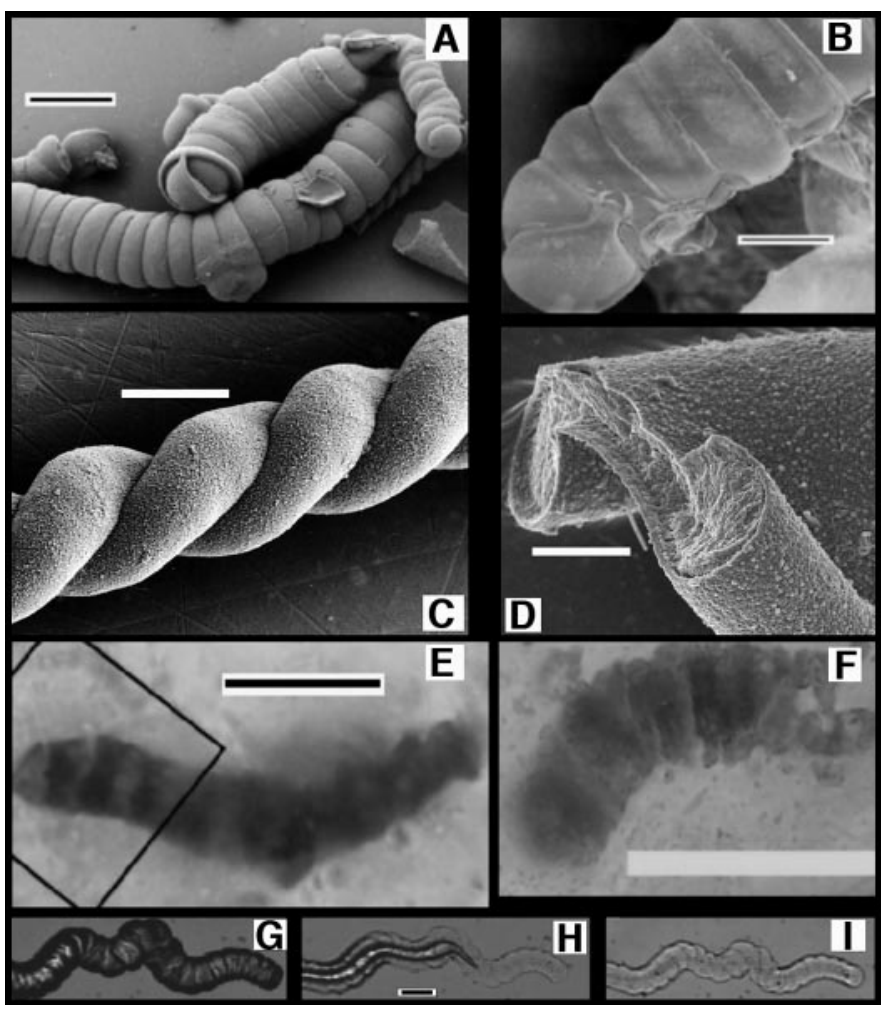

ethanoic acid, leaving a hollow silica membrane whose morphology is that of the original witherite-silica composite. [Adapted with permission from (12).] Scale bars in (A) and (B), $40 \mu \mathrm{m}$; in (C), $10 \mu \mathrm{m}$; in (D), $4 \mu \mathrm{m}$; in (F) to (I), $40 \mu \mathrm{m}[(\mathrm{G})$ and $(\mathrm{I})$ are at the same magnification as $(\mathrm{H})]$. A more detailed sequence is available in movie S3. low helical silica filament of the same size and shape as the untreated filament (Fig. 2, $\mathrm{G}$ to I). These synthetic filaments fulfill the morphological criteria that are currently accepted to confirm the biogenicity of remnants of microbial life $(3,17)$. Evidently, simple biological entities and complex inorganic systems can display common morphological features.

This finding has implications for the search for remnants of primitive life in the early Earth or Mars (18). Microstructures found in chert of the Apex basalt are reputed to be among the oldest microfossils on Earth (3.465 billion years old) (4). Schopf et al. described them as septate, possibly photosynthetic, filamentous cyanobacteria living in shallow water (3-6). In contrast, Brasier et al. suggested that the structures might be abiotic (19). Their reinvestigation of Schopf's samples revealed examples of complex branched filaments, in contrast to the unbranched morphologies mapped by Schopf. In addition, their geological mapping of the area suggested a hydrothermal setting for the putative microbes. Ueno et al. reported chert-hosted microstructures morphologically similar to those of $(5,6)$ but located in the even older (3.49 billion years) Dresser Formation of the Warrawoona Group in the North Pole area. These authors interpreted their microscopic filaments as microfossils (1).

The purported fossils may instead be examples of unconventional abiotic microstructures $(17,19-20)$; our filamentous materials mimic the morphology of Warrawoona microstructures to a high degree (Fig. 2, A to F). Schopf et al. (6) have advanced an additional criterion for biogenicity: the presence of carbonaceous material of biological origin. Chemical evidence for such biogenic material has been adduced from laser Raman microspectroscopy of the Warrawoona microstructures, though this has been disputed (7).

Our experiments suggest an abiotic pathway to the formation of "kerogenous" filaments (filaments containing organic matter that remains insoluble in both acids and organic solvents). For example, kerogenous carbonaceous polymers can be formed by heating simple (plausibly abiotic) organic molecules (21). That process can occur under hydrothermal conditions, given the presence of hydrophobic substrates that act as traps for ambient organic precursors. Our siliceous biomorphs are such traps. For example, immersion of witherite-silica filaments in formaldehyde-phenol mixtures and subsequent heating at $125^{\circ} \mathrm{C}$ for at least 15 hours leaves a brown deposit on the filaments, clearly visible in the optical microscope (Fig. 3, A to C). Laser Raman microspectroscopy confirms the presence of carbonaceous residue in the biomorphic filaments after absorption of the model formaldehyde-phenol mixture and subsequent curing at $500^{\circ} \mathrm{C}(13)$. These spectra 
resemble the kerogen spectra of Warrawoona microfilaments reported by Schopf et al. (6) (Fig. 3D). Clearly, "kerogen" formation can occur in the absence of biological activity. It is therefore important to determine whether the synthetic route we have used to form synthetic kerogenous biomorphs is a plausible model for formation of the Apex microstructures.

The physicochemical environment required for the self-assembly of our biomorphic materials is unusual nowadays. It was, however, geochemically plausible during Archaean times $(22,23)$. Our reaction requires an alkaline medium, silica and carbonate sources, and barium ions, as well as simple organic precursors. Specific horizons in the Warrawoona Group are rich in chert $\left(\mathrm{SiO}_{2}\right)$, barite $\left(\mathrm{BaSO}_{4}\right)$, and carbonate minerals. In particular, the bedded chert-barite Dresser Formation [ $\sim 3.49$ billion years old (24)] contains a unit near the top of the formation, up to $2 \mathrm{~m}$ thick, containing centimeter-scale layers of chert alternating with carbonate and oxide minerals overlying the hydrothermal barite-chert deposit and overlain by wrinkly stromatolite-like mats with numerous small, low-amplitude domes (25). Chert in the lower parts of the formation has been reported to contain numerous microfossils (1), including filamentous examples that are morphologically identical to those reported by Schopf et al. (5), located in the stratigraphically higher Apex basalt. The carbonate layers consist of rhombohedral crystals, which are highly altered to a mixture of dark brown to black iron and manganese oxides. Close inspection of thin sections reveals partial replacement of the original carbonate rhombs by small inclusions of the dark material (Fig. 4). The dark alteration of the rhombs is not associated with veins or the result of obvious subaerial weathering, and is thus interpreted to be an original pre-exhumation feature of the rock. Energy-dispersive $\mathrm{x}$-ray analysis and $\mathrm{x}$-ray diffraction studies confirm the presence of quartz, ankerite, relatively low-iron calcite, goethite, and the manganese oxide romanechite. We interpret the latter two minerals to be ankerite decomposition products, as outlined below.

Our abiotic formation mechanism requires the presence of organics of relatively low molecular weight, such as phenol and formaldehyde. Their occurrence in the Archaean environment is highly plausible, even in the absence of biological activity. Indeed, recent results show that related polycyclic aromatic hydrocarbon compounds (PAHs) can be readily formed from inorganic precursors alone. McCollom has recently demonstrated the formation of a complex mixture of PAHs by heating siderite $\left(\mathrm{FeCO}_{3}\right)$ to $300^{\circ} \mathrm{C}$ in the presence of water vapor (26), with a schematic reaction mechanism as follows: $\mathrm{FeCO}_{3}+\mathrm{H}_{2} \mathrm{O} \rightarrow \mathrm{Fe}_{3} \mathrm{O}_{4}+\mathrm{CO}_{2}+\mathrm{CO}+$ $\mathrm{H}_{2}+$ PAH (aromatic C, O, H organics).

The presence of an iron-bearing carbonate closely related to siderite (that is, ankerite) in the hydrothermal Warrawoona deposit admits the possibility of this reaction. In addition, the replacement texture in
Fig. 3. (A to C) Optical micrographs of the silica-witherite structure before and after exposure to organic species, all taken under identical illumination. (A) Inorganic structure as prepared. (B) Structure after hydrothermal adsorption of organics. (C) Cured material produced by baking the sample that was preexposed to the organic mixture [as in (B)]. Scale bars, $50 \mu \mathrm{m}$. (D) (Upper curve) Raman spectrum of heatcured biomorphs (similar to Fig. 3C) compared with kerogen-like Raman spectrum reported by Schopf et al. (lower curve), collected from a microfilament in the Archean Warrawoona chert [reproduced with permission from fig. $3 \mathrm{H}$ of (6)].

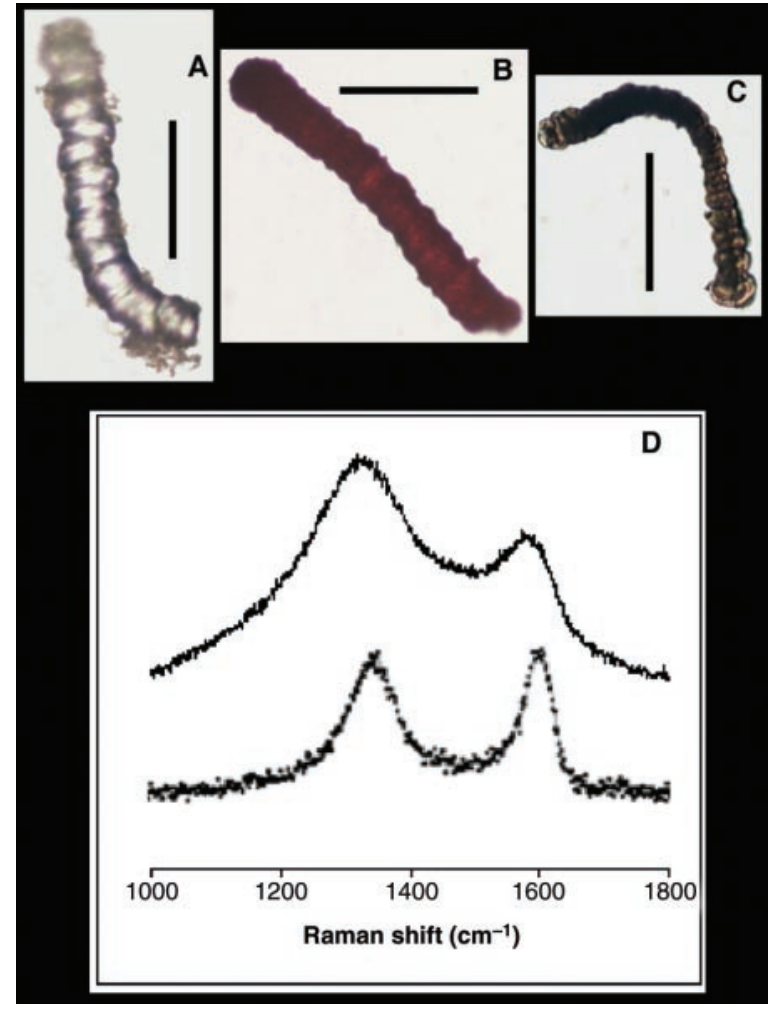

the rhombs (Fig. 4) is consistent with partial decomposition of carbonate and simultaneous formation of (hydr)oxide minerals, analogous to the reaction scheme above. The in situ production of organics based on decomposition of the $\mathrm{Fe}$ (and possibly $\mathrm{Mn}$ ) carbonate fractions, accompanying ankerite decomposition and goethite formation, is thus consistent with the geochemical features of the Warrawoona formation. Assuming inorganic synthesis of these organics, subsequent condensation to form kerogen is possible over geological time. For example, the phenol-formaldehyde condensation described above offers a simple route to kerogen formation. The kinetics of kerogen maturation is a first-order process, with activation energies between 150 and $200 \mathrm{~kJ} / \mathrm{mol}$ (27, 28). Assuming equal kinetics for the aging process in both synthetic and Warrawoona kerogen, the Arrhenius equation implies that an average temperature between $70^{\circ}$ and $125^{\circ} \mathrm{C}$ should have sufficed to produce the kerogen observed in the Warrawoona samples via the condensation pathway described above. This range is consistent with a recent geological investigation that suggests a maximum temperature of $\sim 250^{\circ}$ to $350^{\circ} \mathrm{C}(19)$. Our accelerated synthetic aging protocol is therefore a reasonable mimic of the natural geological process.

The implications of these findings within the context of prebiotic chemistry in alkaline media are manifold. In particular, the assumption of a disjunction between biotic and abiotic morphologies and chemical nature is false for primitive ancient fossil remnants. Furthermore, the complex structural hierarchy of this novel synthetic material, reflecting features of both crystalline and liquid crystalline structures, is of fundamental interest to the materials community (29).

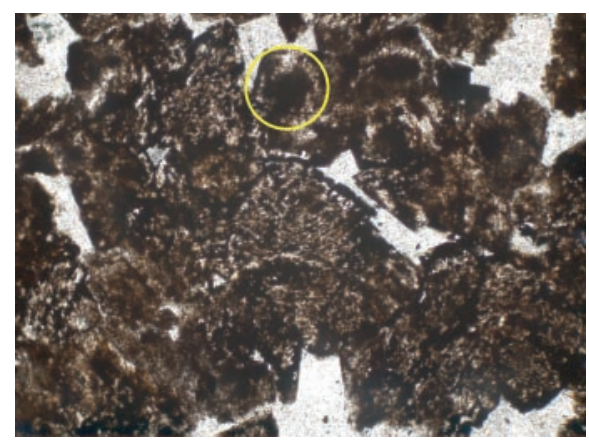

Fig. 4. Plain polarized-light thin-section view of the ankerite-rich layer of a cut slab from the top of the Dresser Formation. The section shows carbonate rhombs in a chert matrix. Note the degraded form of the rhombs and partial replacement by darker material (circled), which may have occurred through inorganic dissociation of ankerite to goethite and romanechite plus calcite. Width of view, $\sim 1.5 \mathrm{~mm}$. 
References and Notes

1. Y. Ueno, Y. Isozaki, H. Yurimoto, S. Maruyama, Int Geol. Rev. 43, 196 (2001).

2. S. M. Awramik, J. W. Schopf, M. R. Walter, Precambrian Res. 20, 357 (1983).

3. J. W. Schopf, M. R. Walter, in Earth's Earliest Biosphere, J. W. Schopf, Ed. (Princeton Univ. Press, Princeton, NJ, 1983), chap. 9.

4. J. W. Schopf, B. M. Packer, Science 237, 70 (1987)

5. J. W. Schopf, Science 260, 640 (1993).

6. J. W. Schopf, A. B. Kudryavtsev, D. G. Agresti, T. J. Wdowiak, A. D. Czaja, Nature 416, 73 (2002).

7. J. D. Pasteris, B. Wopenka, Nature 420, 476 (2002).

8. I. L. Svensson, S. Sjöberg, L.-O. Öhman, J. Chem. Soc. Faraday Trans. 1 82, 3635 (1986).

9. R. M. Garrels, C. L. Christ, Solutions, Minerals and Equilibria (Harper \& Row, New York, 1965).

10. T. Baird et al., Mater. Res. Bull. 27, 1031 (1992)

11. J. M. García-Ruiz, A. Moreno, Anales Química Int. Ed. 93, 1 (1997).

12. J. M. García-Ruiz, A. Carnerup, A. G. Christy, N. J. Welham, S. T. Hyde, Astrobiology 2, 335 (2002).

13. Available as supporting material on Science Online

14. P. G. De Gennes, J. Prost, The Physics of Liquid Crystals (Clarendon, Oxford, 1993).
15. J. H. E. Cartwright, J. M. Garcia-Ruiz, M. L. Novella, F. Otalora, J. Colloid Interface Sci. 256, 351 (2002)

16. H. A. Lowenstam, S. Weiner, On Biomineralization (Oxford Univ. Press, Oxford, 1989).

17. R. Buick, Palaios 5, 441 (1990).

18. B. A. Hofmann, J. D. Farmer, Planet. Space Sci. 48 1077 (2000).

19. M. D. Brasier et al., Nature 416, 76 (2002).

20. P. E. Cloud, K. Morrison, Precambrian Res. 9, 81 (1979).

21. T.-H. Ko, W.-S. Kuo, Y.-H. Chang, Polym. Compos. 21 745 (2000).

22. J. M. Garcia-Ruiz, in Carbonate Sedimentation and Diagenesis in the Evolving Precambrian World, J. P. Grotzinger, N. P. James, Eds. (SEPM Special Publication 67, Society for Sedimentary Geology, Tulsa, OK 2000), pp. 75-89.

23. J. M. Garcia-Ruiz, Geology 26, 843 (1998).

24. R. I. Thorpe, A. H. Hickman, D. W. Davis, J. K. Mortensen, A. F. Trendall, in The Archaean: Terrains, Processes and Metallogeny, J. E. Glover, S. Ho, Eds. (Geology Department and University Extension, The University of Western Australia, 1992), pp. 395-408.

25. M. J. Van Kranendonk, in preparation.

26. T. M. McCollom, Geochim. Cosmochim. Acta 67, 311 (2003)
27. J. M. Hunt, M. D. Lewan, R. J.-C. Hennet, Am. Assoc. Petrol. Geol. Bull. 75, 795 (1991).

28. D. A. Wood, Am. Assoc. Petrol. Geol. Bull. 72, 115 (1988).

29. H. Yang, N. Coombs, G. A. Ozin, Nature 386, 692 (1997).

30. We thank J. Doherty [formerly Applied Maths, Australian National University (ANU); now at Australian Capital Territory Government Analytical Laboratory] for her research contributions; also F. Brink, C. Huang and S. Stowe of the Electron Microscopy Unit, ANU; U. Troitzsch (Department of Geology, ANU); and V. OtienoAlego (formerly Corrosion and Spectrochemistry Laboratory, University of Canberra; now Australian Federal Police Forensic Laboratory) for their assistance and advice. Published with permission of the director of the Geological Survey of Western Australia.

Supporting Online Material

www.sciencemag.org/cgi/content/full/302/5648/1194/ DC1

Materials and Methods

Figs. S1 to S3

Movies S1 to S4

Table S1

6 August 2003; accepted 6 October 2003

\section{Intraslab Earthquakes: Dehydration of the Cascadia Slab}

\author{
Leiph A. Preston, ${ }^{1 *}$ Kenneth C. Creager, ${ }^{1}$ Robert S. Crosson, ${ }^{1}$ \\ Thomas M. Brocher, ${ }^{2}$ Anne M. Trehu ${ }^{3}$
}

\begin{abstract}
We simultaneously invert travel times of refracted and wide-angle reflected waves for three-dimensional compressional-wave velocity structure, earthquake locations, and reflector geometry in northwest Washington state. The reflector, interpreted to be the crust-mantle boundary (Moho) of the subducting Juan de Fuca plate, separates intraslab earthquakes into two groups, permitting a new understanding of the origins of intraslab earthquakes in Cascadia. Earthquakes up-dip of the Moho's 45-kilometer depth contour occur below the reflector, in the subducted oceanic mantle, consistent with serpentinite dehydration; earthquakes located down-dip occur primarily within the subducted crust, consistent with the basalt-to-eclogite transformation.
\end{abstract}

The most damaging earthquakes in western Washington have been intraslab events, also known as Wadati-Benioff earthquakes. These include earthquakes in 1949, 1965, and, most recently, the 2001 moment magnitude $\left(M_{\mathrm{w}}\right)$ 6.8 Nisqually event. Although megathrust earthquakes are typically larger in magnitude, intraslab events can be more damaging because they often occur directly beneath population centers, may have shorter recurrence intervals (as in Washington), and tend to have larger seismic energy-to-moment ratios (1) than megathrust events have.

The physical mechanisms responsible for intraslab earthquakes in the depth range 30 to $200 \mathrm{~km}$ have been debated for de-

${ }^{1}$ Department of Earth and Space Sciences, University of Washington, Seattle, WA 98195, USA. '2U.S. Geological Survey, Menlo Park, CA 94025, USA. ${ }^{3}$ College of Oceanic and Atmospheric Sciences, Oregon State University, Corvallis, OR 97331, USA.

*To whom correspondence should be addressed. Email: preston@ess.washington.edu cades. A prominent theory, dehydration embrittlement, asserts that volatiles released during metamorphic dehydration reactions reduce the effective normal stresses across faults, allowing slip $(2,3)$. In light of the damaging 2001 Nisqually $\left(M_{\mathrm{w}} 6.8\right)$, 2001 Geiyo, Japan $\left(M_{\mathrm{w}} 6.7\right)$, and $2001 \mathrm{El}$ Salvador $\left(M_{\mathrm{w}}\right.$ 7.7) intraslab earthquakes, gaining physical insight into the mechanics controlling these earthquakes is important for earthquake hazard estimates and mitigation efforts. The 1998 Seismic Hazards Investigation in Puget Sound (Wet SHIPS) experiment (4) provided an opportunity to investigate the high-resolution structure of the subducting plate in northwest Washington and southwest British Columbia (5).

The Juan de Fuca plate is relatively young and warm (maximum age $\sim 10$ million years), subducting obliquely at about $40 \mathrm{~mm} /$ year northeastward under Washington and Oregon. Active intraslab seismicity extends to $60-\mathrm{km}$ depth, with some small events reaching depths as great as $100 \mathrm{~km}(6)$.
The data for the structural inversion consist of 90,000 first-arrival travel times from the Wet SHIPS, Dry SHIPS, western Cascades, and southwest Washington experiments (fig. S1) (7, 8); 27,000 first-arrival times from 1400 local earthquakes [200 of which are intraslab events (9)]; and 1200 secondary arrivals from the Wet SHIPS experiment that are consistent in slowness and travel time with reflections from the Juan de Fuca slab. We developed a nonlinear iterative inversion scheme that simultaneously inverts these travel times for earthquake locations, three-dimensional (3D) velocity structure, and reflector geometry (10). A wellknown trade-off exists between reflector depth and velocity structure. By including independent first-arrival information, we reduce this trade-off and extract reliable reflector depths. A smooth velocity model is regularized by minimizing second-order spatial derivatives of the velocity structure and reflector surface. Travel times of first arrivals are calculated using the Vidale-Hole $(11,12)$ 3D finite-difference code. Theoretical reflected bounce points and travel times are determined by summing calculated travel times from the source and receiver to points on the reflector surface and determining the position and time corresponding to the minimum summed time according to Fermat's principle. Reflected rays are then independently traced from the bounce point to the source and to the receiver. Reflector geometry and the 3D velocity model are each adjusted to fit the times of reflected waves. This nonlinear procedure converges stably after 10 iterations. The final model gives root mean square travel-time residuals of $0.09,0.12$, and $0.08 \mathrm{~s}$ for the active-source, earthquake, and reflection data, respectively, amounting to variance reductions of 98 and $91 \%$ for the active-source and earthquake travel times, respectively, relative to the standard regional $1 \mathrm{D}$ velocity model (13). 